\title{
TAK1 regulates hepatic cell survival and carcinogenesis
}

\author{
Yoon Seok Roh $\cdot$ Jingyi Song $\cdot$ Ekihiro Seki
}

Received: 4 December 2013/Accepted: 24 December 2013/Published online: 21 January 2014

(C) Springer Japan 2014

\begin{abstract}
TGF- $\beta$-activated kinase 1 (TAK1 or MAP3K7) is an intracellular hub molecule that regulates both nuclear factor- $\kappa \mathrm{B}(\mathrm{NF}-\mathrm{\kappa B})$ and mitogen-activated protein kinase (MAPK) signaling pathways that play key roles in development, cell survival, immune response, metabolism, and carcinogenesis. TAK1 activity is tightly regulated by its binding proteins, TAB1 and TAB2/TAB3, as well as by post-translational modification including ubiquitination and phosphorylation. Accumulating evidence demonstrates that TAK1 plays a role in tumor initiation, progression, and metastasis as a tumor prompter or tumor suppressor. An understanding of the role of TAK1 in liver physiology and diseases is required for the development of therapeutic agencies targeting TAK1. In this review, we highlight the activation mechanism and pathophysiological roles of TAK1 in the liver.
\end{abstract}

Keywords HCC $\cdot$ NF- $\mathrm{KB} \cdot \mathrm{TGF}-\beta \cdot$ Liver cancer . Apoptosis

\section{Introduction}

TAK1 is a serine/threonine kinase that was identified by a cDNA library screening and protein-fragment complementation assay in yeast for its ability to substitute for the MAPKKK Ste11p in the yeast pheromone-induced MAPK pathway. Thereby, TAK1 was validated as a member of the MAPKKK family (MAP3K7) [1]. TAK1 is a critical mediator of the inducible transcription factors, such as

Y. S. Roh · J. Song · E. Seki $(\bowtie)$

Department of Medicine, University of California, San Diego,

School of Medicine, La Jolla, CA 92093, USA

e-mail: ekseki@ucsd.edu nuclear factor- $\kappa \mathrm{B}(\mathrm{NF}-\kappa \mathrm{B})$ and c-Jun N-terminal kinases (JNKs), that contribute to embryonic development, innate immunity, and cell survival; therefore, TAK1 plays a crucial role in the regulation of genes involved in cell homeostasis. Recent reports demonstrate that TAK1 acts as a tumor suppressor in the liver and prostate [2, 3]. In contrast, the inhibition of TAK1 activity induces cancer cell death, suggesting that TAK1 may be an effective target for cancer treatment $[4,5]$. Therefore, understanding the regulation of TAK1 activity and its functions are important for the development of cancer therapies targeting TAK1.

\section{Regulation of TAK1 activity}

TAK1 forms a complex with its adaptor proteins, TAB1, $\mathrm{TAB} 2$, and TAB3. TAB1 constitutively interacts with TAK1 and participates in the autophosphorylation of TAK1 that is essential for TAK1 kinase activity [6]. In contrast to $\mathrm{TAB} 1, \mathrm{TAB} 2$ and $\mathrm{TAB} 3$ are structurally related and share $48 \%$ of their amino acid sequences. In contrast to TAB1, which binds to the $\mathrm{N}$-terminal kinase domain of TAK1, TAB2 and TAB3 associate with the C-terminal region of TAK1 (Fig. 1) [7, 8]. Upon stimulation with cytokines such as IL-1 $\beta$, TAB2 induces translocation from the cell membrane to the cytosol. TAB2 then associates TAK1 with ubiquitinated TRAF6, thereby mediating the activation of TAK1. The genetic deletion of TAB1, TAB2, or TAK1 results in embryonic lethality; $\mathrm{TAB} 1^{-1-}$ mice die during a late stage of gestation with defects in both cardiac and lung development. While TAB 2 and TAB3 are suggested to function redundantly, $\mathrm{TAB} 2^{-/-}$mice are embryonically lethal with abnormalities in the liver [9-11]. These findings suggest that TAK1 and its associated proteins have distinct regulatory mechanisms and 
Fig. 1 The domain structure of TAK1. The TAK1 protein contains a TAB1-binding region and kinase domain in the $\mathrm{N}$-terminus and a TAB2/TAB3 binding domain in the C-terminus

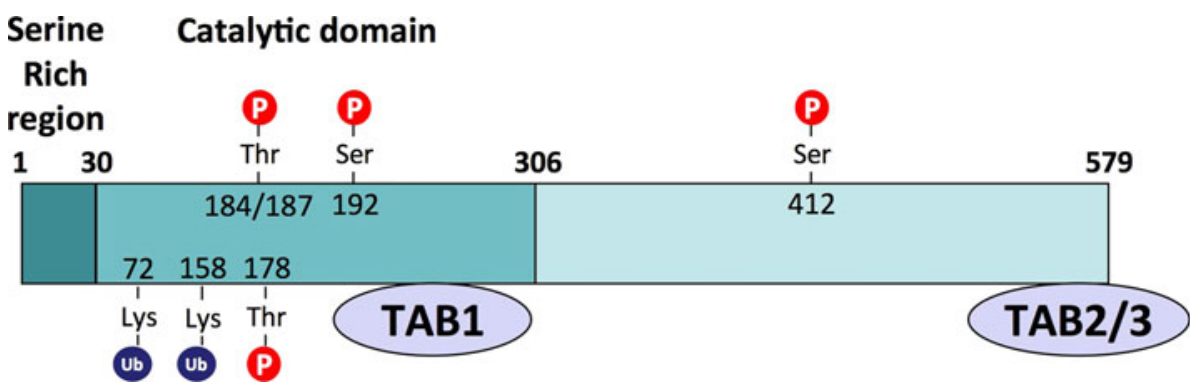

functions that are not redundant in the regulation of TAK1 activity.

Multiple post-translational modifications of TAK1 and TABs control TAK1 kinase activity. Although the phosphorylation of Thr-187 is crucial for TAK1 kinase activity in the TNF receptor (TNFR) signaling, ubiquitination and methylation are also important for the regulation of TAK1 activity [12]. Furthermore, the O-linked $\beta$ - $N$-acetylglucosaminylation (O-GlcNAcylation) of TAB1 at Ser-395 is associated with the full activation of TAK1 [13].

TAK 1 is activated by the signaling of TGF- $\beta$ receptor, IL-1 receptor (IL-1R), Toll-like receptor (TLR), type 1 TNFR (TNFR1), T cell receptor (TCR), and B cell receptor (BCR). These signalings, except for the TNFR signaling, induce the association of TNF receptor-associated factor (TRAF) 6 with TAK1 to activate IKK and JNK/p38 MAP kinases [14-16]. In contrast, TNFR1 signaling utilizes TRAF2, TARF5, and RIP1 to propagate signaling to activate TAK1 [17]. In TNFR signaling, TNF- $\alpha$ trimer binding to trimerized TNFR1 induces the recruitment of adaptor proteins TRADD, cIAPs, RIP1 kinase, and E3-ubiquitine ligases TRAF2 and TRAF5, to the intracellular domain of TNFR1. TRAF2/5, cIAPs, and Ubc13 promote the K63 polyubiquitination of RIP1 (Fig. 2) [18, 19]. Then, the TAB2/TAB3 molecule of the TAK1-TAB1-TAB2/TAB3 complex is recruited to K63 polyubiquitin chains of RIP1. TAB1 is involved in TAK1 autophosphorylation at Thr184 and Thr187, and the binding of RIP1 polyubiquitin chains to the TAK1 complex through TAB2 or TAB3 is required for TAK1 activation. The $N F-\kappa B$ essential modulator (NEMO) of the IKK complex (IKK $\alpha / \mathrm{IKK} \beta / \mathrm{NEMO}$ ) is also recruited to RIP1 K63 polyubiquitin chains (Fig. 2). Unlike K48 polyubiquitination chains involved in the proteasomal degradation, K63 polyubiquitin chains promote signaling by serving as molecular scaffolds. Activated TAK1 phosphorylates IKK $\beta$, which then induces $\mathrm{I} \kappa \mathrm{B} \alpha$ phosphorylation and K48 ubiquitination followed by proteasomal degradation of $\mathrm{I} \kappa \mathrm{B} \alpha$. The freed $\mathrm{NF}-\kappa \mathrm{B}$ then nuclear-translocates itself and binds to $\kappa \mathrm{B}$ motifs of the DNA. NF- $\kappa \mathrm{B}$ activation leads to the expression of antiapoptotic molecules, such as c-FLIP and anti-oxidant
SOD2 (Fig. 2). These NF- $\kappa \mathrm{B}$ inducible molecules prevent the caspase-dependent apoptotic pathway and antagonize ROS production that promotes the JNK-dependent proapoptotic pathway [19]. TLR, IL-1, and TGF $\beta$ signaling all utilize another E3-ubiquitine ligase, TRAF6, to build K63 polyubiquitin chains on TRAF6 in the presence of Ubc13. The K63 polyubiquitin chains bind to TAK1 and IKK complexes for activation of these complexes, followed by NF- $\kappa \mathrm{B}$ activation (Fig. 2) [15, 18, 20, 21].

$\mathrm{TAB}^{-1-}$ cells activate $\mathrm{JNK}$, but not $\mathrm{NF}-\kappa \mathrm{B}$, because the TAK1 complex binds to the polyubiquitin chains of RIP1/TRAF2 or TRAF6 through TAB2, and the activation of IKK requires both TAK1 complex and IKK complex binding to polyubiquitination chains [22]. Therefore, it is likely that the $\mathrm{NF}-\kappa \mathrm{B}$ pathway is activated in an ubiquitination-dependent manner, but the MAPK pathway is activated in a phosphorylation-dependent manner rather than a ubiquitination-dependent manner.

Deactivation of TAK1 is also an important molecular mechanism in the regulation of TAK1 activity. Thr-187, a crucial autophosphorylation site can be rapidly dephosphorylated by protein phosphatase 6 (PP6) [23]. The activation of PP6 requires the binding to K63 polyubiquitin chains through TAB2. Therefore, in the absence of TAB2, PP6 cannot dephosphorylate TAK1, thereby prolonging TAK1 and JNK phosphorylation (Fig. 3) [22]. Thus, TAB2 controls not only the activation of TAK1, but also the deactivation of TAK1 through PP6. Furthermore, the feedback loop by p38 contributes to TAK1 deactivation. p38 $\alpha$ phosphorylates TAB1 at Ser-423 and Thr-431, which promotes TAK1 dephosphorylation [24]. Deubiquitinases CYLD and A20 form complexes with the E3 ubiquitin ligase ITCH that cleave K63-linked polyubiquitin chains [25], and act together to terminate TAK1-mediated inflammatory signaling [26]. The CYLD-ITCH or A20ITCH complex cleaves K63-linked polyubiquitin chains on activated TAK1 and catalyzes K48-linked ubiquitination to terminate TAK1-mediated $\mathrm{NF}-\kappa \mathrm{B}$ activation through proteosomal degradation of TAK1 [26, 27]. Deubiquitinases A20 and Cezanne, but not CYLD, are induced by NF- $\kappa$ B to negatively regulate TAK1 and $\mathrm{NF}-\kappa \mathrm{B}$ activity by removing K63 polyubiquitin chains [28]. 
Fig. 2 Activation of TAK1 signaling. (1) Binding of TNF to the TNFR type 1 leads to the formation of a complex comprising TRADD, RIP1, TRAF2/5, and Ubc13. K63 ubiquitination of RIP1 recruits and activates TAK1 through TAB2. (2) IL-1R, TLR, TGF- $\beta$, TCR, and BCR utilize TRAF6 and Ubc13 to create K63 polyubiquitin chains that recruit TAB2 of the TAK1 complex. (3) TAK1 phosphorylates and activates the IKK complex, leading to the phosphorylation, ubiquitination, and degradation of $\mathrm{I} \kappa \mathrm{B} \alpha$. The freed NF- $\kappa \mathrm{B}$ is activated and translocated into the nucleus. (4) MAPK pathways are activated in a phosphorylation-dependent manner. (5) DNA double-strand breaks (DSB) induce the sumoylation and monoubiquitination of NEMOs that interact with ATM. The NEMO-ATM complex promotes K63

polyubiquitination by Ubc13, which in turn activates TAK1 and IKK

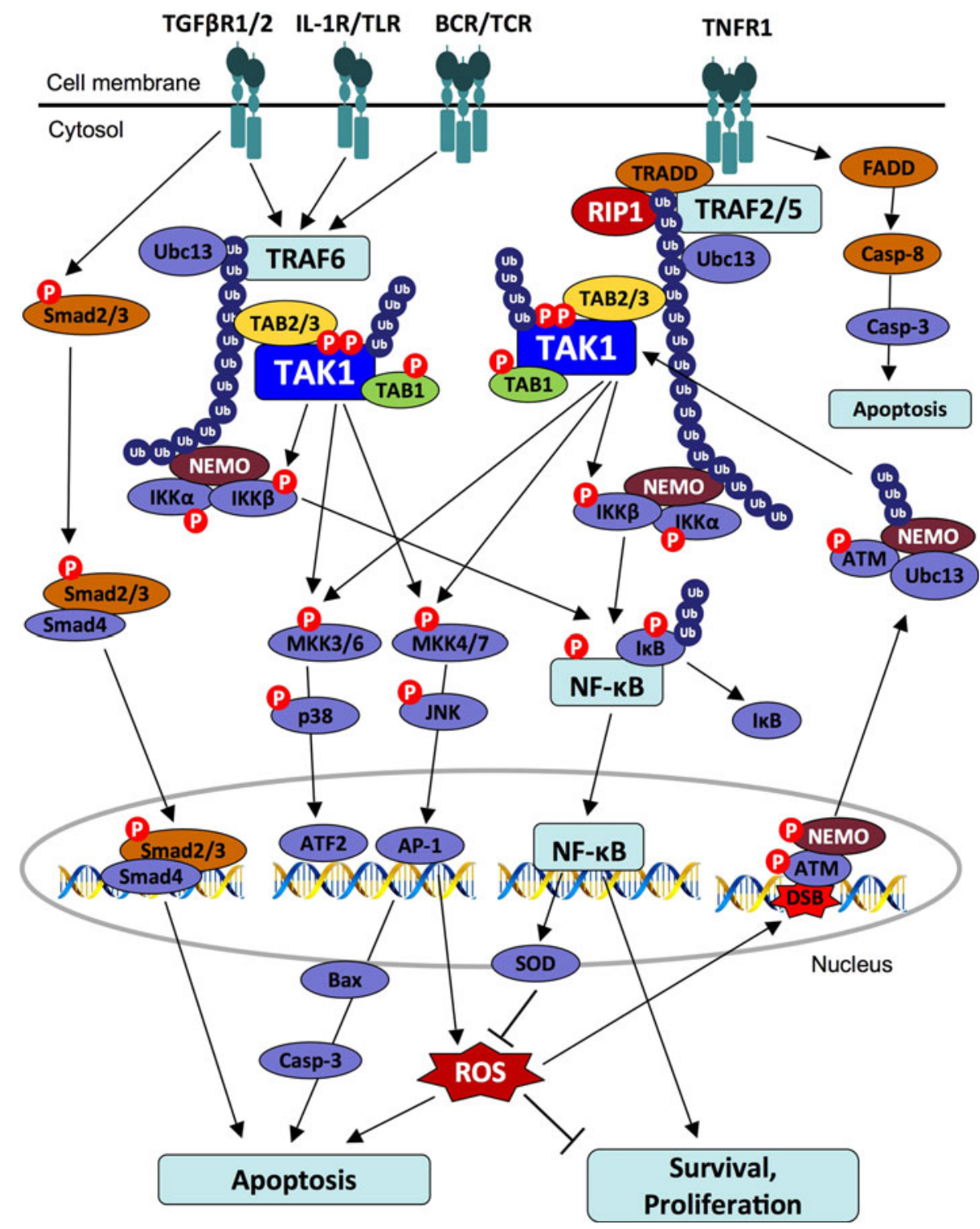

\section{TAK1 and TGF- $\beta$ signaling}

TGF- $\beta$ family signaling contributes to a variety of cellular and tissue pathophysiology, including cell survival, proliferation, differentiation, embryonic development, inflammation, and carcinogenesis. TGF- $\beta$ binds to the type II TGF$\beta$ receptor and subsequently recruits and phosphorylates type I TGF- $\beta$ receptor kinase. Phosphorylated type I TGF- $\beta$ receptor then activates a Smad-dependent canonical pathway and Smad-independent, non-canonical, TAK1-dependent pathway. In mouse hepatocytes and cardiomyocytes, TAK1 is rapidly activated within $5-10$ min after TGF- $\beta$ stimulation and mediates the activation of $\mathrm{p} 38$ and JNK [20, 29-31]. TAK1-deficient MEFs have reduced the TGF- $\beta$ induced activation of NF- $\kappa B$ and JNK [9]. Intriguingly, the phenotype of conditional TAK1 knockouts resembles that of the ALK I (TGF- $\beta$ type I receptor) knockouts, showing abnormal vascular development [32] and suggesting the dominant functions of TAK 1 in TGF- $\beta$ signaling. Recently, the mechanism by which TGF- $\beta$ mediates TAK 1 activation has been elucidated. When TGF- $\beta$ binds to its receptor complex, type I and type II TGF- $\beta$ receptors recruit TRAF6 and bind to the TRAF homologous domain at the $\mathrm{C}$-terminus of TRAF6 [30,33]. Ubiquitinated TRAF6 interacts physically with TAK1 by K63-linked polyubiquitin chains, leading to the triggering of TAK1 activation [20,33]. However, it is claimed that the unanchored $\mathrm{K} 63$ polyubiquitin chain can directly activate TAK1 due to the fact that TAK1 activity correlates with the polyubiquitin chains and not with TRAF6 [34]. K158 on TAK1 is also ubiquitinated by TRAF6, which is crucial in TAK1-mediated IKK, JNK, and p38 activation by TGF- $\beta$ [35]. 
Fig. 3 Mechanism of TAK1 inactivation. A20, CYLD, and ITCH deubiquitinate TRAF6 and RIP1. Cezanne deubiquitinates RIP1.

Activation of PP6 requires

TAB2 and binding to K63 polyubiquitin chains. PP6 dephosphorylates TAK1. TAK1 and EGFR activate p38, which phosphorylates TAB1, which in turn dephosphorylates TAK1 to negatively regulate TAK1 activity

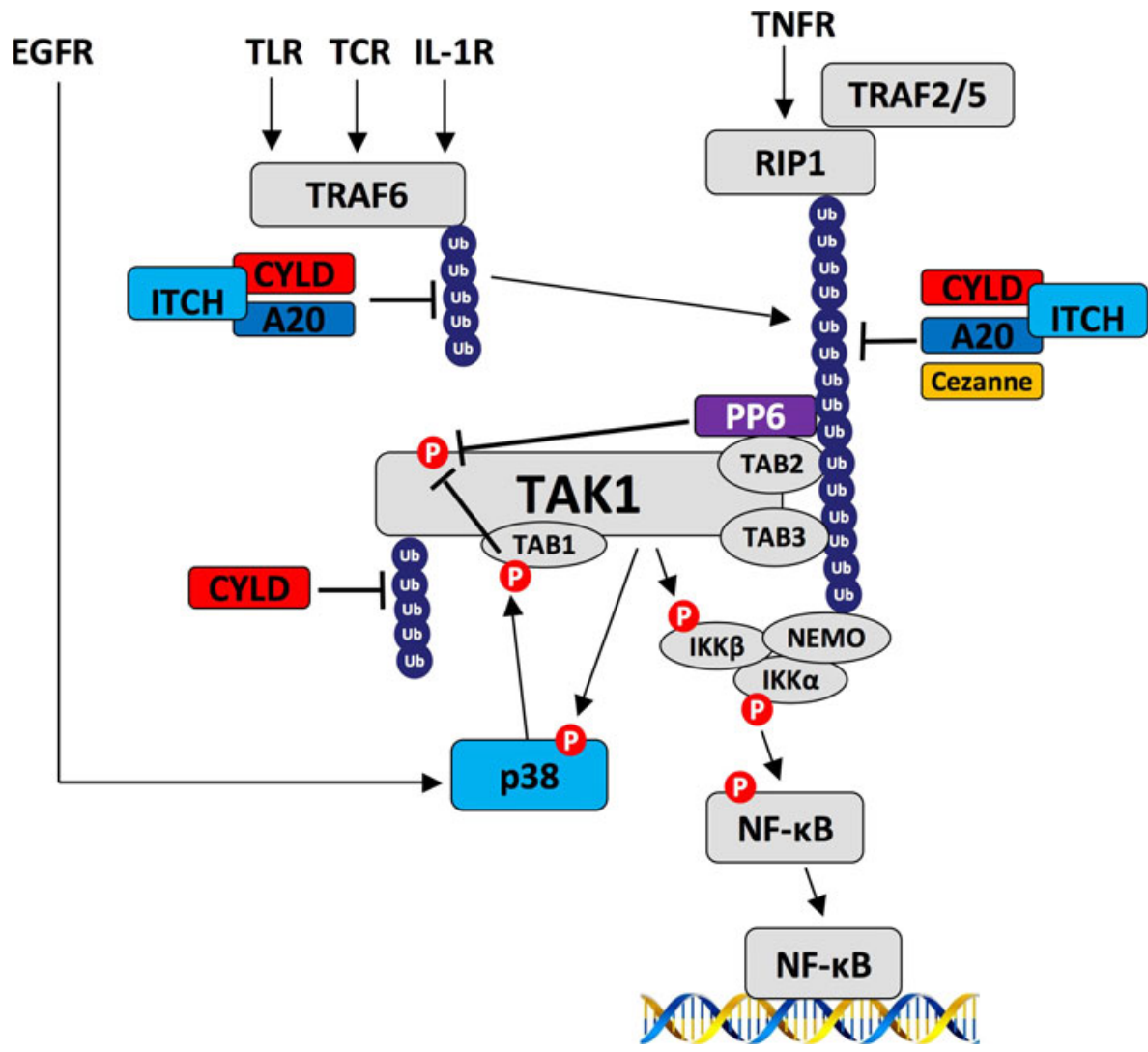

\section{TAK1 and DNA damage response}

Novel function of TAK1 in the DNA damage response has recently been discovered. In cells, DNA repair and cell cycle checkpoint pathways are tightly coordinated. DNA damage by genotoxicity or ionizing radiation arrests cell growth temporarily for DNA repair; severe DNA damage may initiate apoptosis. As a response to genotoxic stress such as chemotherapy and radiotherapy, cells activate NF$\kappa \mathrm{B}$, which leads to cell survival and tumor resistance. Accumulating evidence has shown that TAK1 is required for the activation of NF- $\mathrm{KB}$ in response to genotoxicity [36-38]. However, in the context of the magnitude and duration of TAK1 activity, activation by either ionizing radiation or drugs such as doxorubicin may activate different signaling pathways [37]. In response to genotoxic drugs, ataxia-telangiectasia-mutated (ATM) kinase is mobilized to the nucleus and assembles an ATM-NEMOUbc13 complex that covalently attaches K63-linked polyubiquitin chains to the ELKS (a protein rich in glutamate, leucine, lysine, and serine) adaptor (Fig. 2) [36]. Upon the binding of TAB2 or TAB3 to ubiquitinated ELKS, TAK1 activates the downstream signaling [36]. An ATMTRAF6-Ubc13 module stimulates TAB2-dependent TAK1 phosphorylation in response to ionizing radiation (Fig. 2) [38]. In addition, another E3 ligase, known as linear ubiquitin chain assembly complex (LUBAC), has been demonstrated to be important for the genotoxic activation of TAK1 [39]. Collectively, DNA damage activates TAK1 by similar mechanisms to those found in the IL-1/TNF- $\alpha$ / TLR pathways, via K63 polyubiquitin chains.

\section{Role of TAK1 in hepatocytes}

We and others have demonstrated the critical function of hepatic TAK1 in liver physiology and pathology $[2,40]$. At 1 month of age, mice with TAK1 deletion in hepatocytes develop spontaneous hepatocyte death, compensatory hepatocyte proliferation, hepatic inflammation, and perisinusoidal fibrosis. Older mice develop multiple liver tumors and increase the expression of oncogenic molecules including $\alpha$-fetoprotein [2].

In addition to the spontaneous hepatocyte death caused by TAK1 deletion, $\mathrm{TAK} 1^{-1-}$ hepatocytes increase their sensitivity to TNF- $\alpha$-induced cell death, speculated to be the result of the abrogation of prosurvival NF- $\kappa B$ activity in these cells $[2,9,41,42]$. Additional deletion of TNFR1 in hepatocyte-specific, TAK1-deficient (TAK1 $\Delta \mathrm{Hep})$ mice rescues hepatocytes from cell death $[2,43]$. This indicates that TNFR signaling is responsible for the spontaneous hepatocyte death, liver inflammation, and fibrosis in 
TAK1 $\Delta$ Hep mice. Although TNF- $\alpha$-induced JNK activation is abolished in TAK $1^{-1-}$ hepatocytes, JNK is spontaneously activated in the livers of TAK $1 \Delta \mathrm{Hep}$ mice, in which the cells responsible for activated JNK include both the hepatocytes and liver non-parenchymal cells [2]. In non-parenchymal cells that express intact TAK1 protein, JNK can be activated by several cytokine stimuli, including TNF- $\alpha$ and IL-1 $\beta$, through TAK1. However, it is speculated that in hepatocytes, JNK is activated independently of TAK1 through other cytokine signaling or ROS.

Inhibition of NF- $\mathrm{KB}$ activation by NEMO deletion sensitizes hepatocytes to lipopolysaccharide (LPS)-induced apoptosis that is TNF- $\alpha$-dependent [44]. TAK1 $\Delta$ Hep mice also show massive liver cell apoptosis and liver injury after LPS treatment. The specific deletion of TAK1 in liver parenchymal cells (hepatocytes and cholangiocytes) induces hepatocyte dysplasia and the early-onset of hepatocarcinogenesis, correlating with the formations of biliary ductopenia, cholestasis, and liver fibrosis. Surprisingly, Luedde and colleagues demonstrated the reduction of cancer formation in mice with a liver-specific deletion of NEMO in addition to TAK1, showing this liver phenotype to be dependent on NEMO, but not on NF- $\mathrm{BB}$ [40]. TAK1deleted livers show increased expression of FOXO3a, whereas additional NEMO deletion decreases FOXO3a expression, suggesting that NEMO-dependent FOXO3a expression may contribute to NEMO-dependent carcinogenesis in liver-specific TAK1-deleted mice [40].
Confirmation of this speculation by the genetic deletion of FOXO3a is much desired. Further investigation is necessary to clarify the mechanisms of NEMO-dependent carcinogenesis in TAK1 $\Delta$ Hep mice.

Hepatocytes deficient in TAK1 abrogated the TGF- $\beta$ mediated activation of $\mathrm{p} 38$, JNK, and NF- $\mathrm{KB}$. In contrast, TGF- $\beta$-mediated cell death and phosphorylation of Smad2/ 3 were enhanced [45]. In addition, blocking the Smad pathway inhibited the TGF- $\beta$-induced death of $\mathrm{TAK}^{-1-}$ hepatocytes. Accordingly, the additional deletion of Smad4, a co-Smad of Smad2 and Smad3, inhibited the development of spontaneous liver injury, inflammation, fibrosis, and HCC in TAK1 $\Delta$ Hep mice [45]. These findings indicate that TAK1 positively regulates the prosurvival $\mathrm{NF}-\kappa \mathrm{B}$ pathway and negatively regulates the overactivation of TGF- $\beta$-mediated Smad $2 / 3$ that promotes hepatocyte death (Fig. 4). We also found that TGF- $\beta$ induces the CTGF expression in hepatocytes that is associated with the formation of spontaneous liver fibrosis in TAK1 $\Delta \mathrm{Hep}$ mice. This suggests that not only hepatic stellate cells, but also hepatocytes, contribute to TGF- $\beta$-mediated liver fibrosis. TGF- $\beta$ signaling promotes hepatocyte death and compensatory proliferation during the early course of hepatocarcinogenesis. In contrast, in the advanced stage of hepatocellular carcinoma, TGF- $\beta$ signaling participates in the expression of anti-apoptotic molecules, such as Bcl-xL, chemotactic mediator CTGF, as well as tumor-related molecules such as $\beta$-catenin, YAP1, and WISP1,
Fig. 4 TGF- $\beta$ signaling promotes HCC development in TAK1 $\Delta$ Hep mice. (1) In young hepatocyte-specific TAK1deficient (TAK1 $\Delta$ Hep) mice, $\mathrm{NF}-\kappa \mathrm{B}$ activation is inhibited, while TGF- $\beta$-induced Smad $2 / 3$ activation is enhanced, which promotes hepatocyte death and compensatory proliferation. (2) During hepatocyte

transformation into malignant cells, the TGF- $\beta$-Smad pathway changes its properties, which leads to the upregulation of procarcinogenic, pro-angiogenic, pro-fibrotic, and anti-apoptotic molecules that maintain tumor cell survival and growth

\section{Early phase - TAK1 $1 \mathrm{Hep}$ mouse}

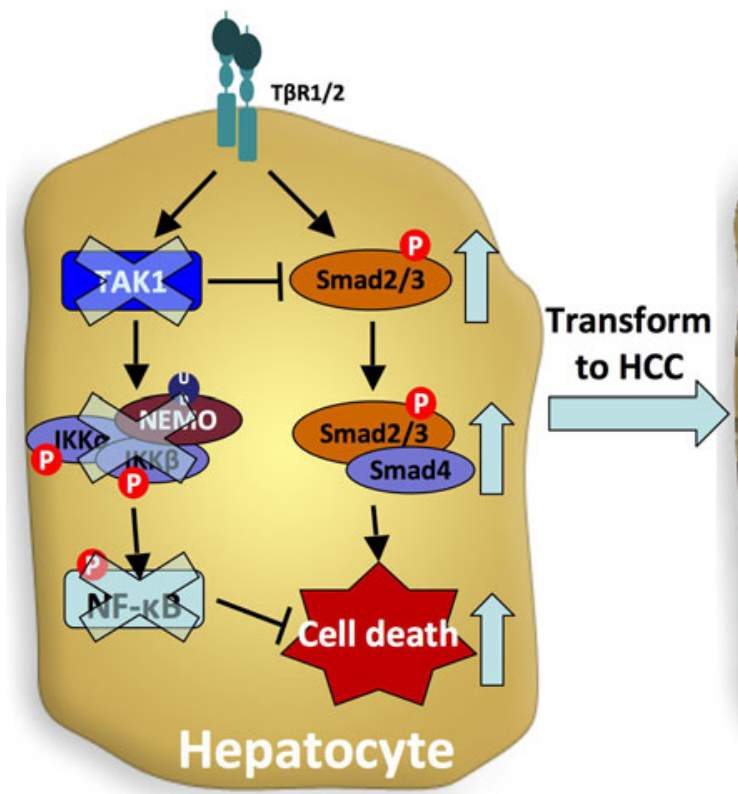

Hepatocyte death

\section{Late phase - TAK1 $\Delta$ Hep mouse}

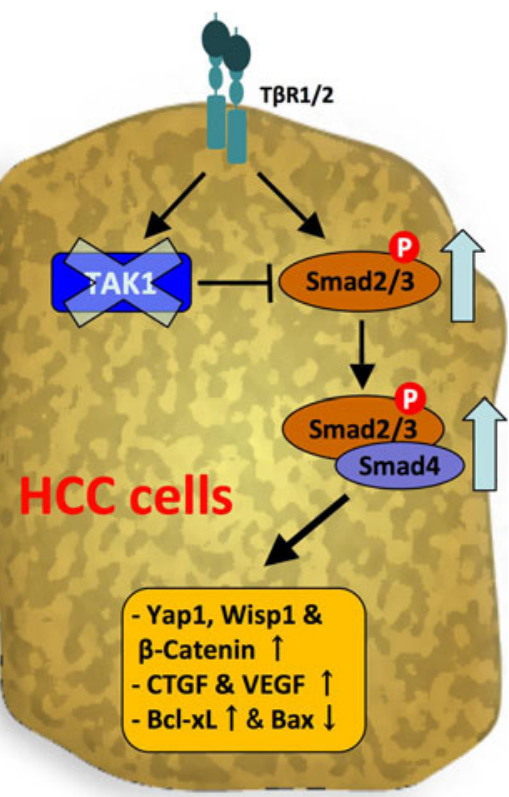

Tumor promotion 
suggesting that TGF- $\beta$-mediated functions change from pro-apoptotic to anti-apoptotic and pro-carcinogenic when cells transform to malignant cells (Fig. 4). Recently, our research group has reported that hepatocyte TGF- $\beta$ signaling contributes to the development of non-alcoholic steatohepatitis through the promotion of hepatocyte death and lipid accumulation in a Smad- and ROS-dependent manner [46]. Although TGF- $\beta$ does not induce cell death in normal hepatocytes, lipid accumulated hepatocytes are susceptible to TGF- $\beta$-mediated cell death. In lipid accumulated hepatocytes, the TAK1-NF- $\kappa \mathrm{B}$ signal acts to prevent cell death and Smad activation. Moreover, ROS production is associated with TGF- $\beta$-mediated cell death. Evidence suggests that TGF- $\beta$-induced Smad and TAK1 activation may act reciprocally in a hepatic stress signaling, resulting in hepatocyte death, inflammation, steatohepatitis, fibrosis, and carcinogenesis [45].

\section{Role of TAK1 in extrahepatic cells}

TAK1 deletion in skin leads to massive keratinocyte death and severe skin inflammation by postnatal day $6-8$. TAK1 deletion increases the sensitivity to TNF- $\alpha$-induced apoptosis in keratinocytes. These findings indicate that the TAK1-IKK-NF- $\mathrm{BB}$ pathway is a critical regulator of epidermis integrity and is essential for the protection of skin from TNF- $\alpha$-induced cell death [47]. Since the deletion of TAK1 in keratinocytes causes ROS accumulation, TAK1 prevents ROS production, which promotes epithelial cell death and inflammation $[48,49]$. TAK $1^{-1-}$ keratinocytes also display exacerbated TNF- $\alpha$-induced JNK activation, and inhibition of ROS by butylated hydroxyanisole (BHA) suppresses TNF- $\alpha$-induced cell death and JNK activation. Moreover, the JNK inhibitor was shown to suppress TNF$\alpha$-induced cell death in TAK $1^{-1-}$ keratinocytes, indicating that JNK activation by ROS is responsible for TNF- $\alpha$ induced cell death in TAK $1^{-1-}$ keratinocytes [49]. Similar to $\mathrm{TAK} 1^{-1-}$ keratinocytes, the deletion of both TAB 1 and $\mathrm{TAB} 2$ in keratinocytes results in the accumulation of ROS, cell death, and inflammation in skin [50].

TAK1 deletion in the intestinal epithelium also causes spontaneous intestinal inflammation and apoptosis, resulting in fatality merely 1 day after birth. Rates of TNF- $\alpha-$ induced intestinal apoptosis and inflammation significantly increase in intestine-specific TAK1 deficient (TAK1 $\Delta$ Int) mice. The additional deletion of TNFR1 attenuates, but does not completely inhibit, intestinal apoptosis and inflammation in TAK $1 \Delta$ Int mice, demonstrating that TNFR signaling partially mediates damage, but that the TNFR-independent mechanism is also important for the intestinal damage in TAK1 $\Delta$ Int mice [51]. Mechanistically, TAK1 signaling regulates the integrity of the intestinal epithelium through a key antioxidant transcription factor, NF-E2-related factor 2 [48]. Recently, several reports suggest that TAK1 protects cells from TNF-induced necroptosis by preventing RIP1/RIP3-dependent ROS production and caspase- 8 activation $[52,53]$. These findings suggest that TAK1 mediates the prosurvival signaling that protects cells from apoptosis induced by inflammatory cytokines and ROS.

TAK1 deletion in cartilage leads to the abnormal development of cartilage, and severe postnatal growth retardation culminating in death by 2-3 weeks of age [54]. Cartilage-specific $\mathrm{TAK}^{-/-}$mice display severe chondrodysplasia, impaired formation of secondary centers of ossification, and joint abnormalities including elbow dislocation and tarsal fusion. Of note, bone morphogenic protein receptor (BMPR) signaling is impaired in TAK $1^{-1-}$ chondrocytes, resulting in reduced phosphorylation of Smad1/5/8 and p38/JNK/ERK MAP kinases.

Through endothelial-specific deletions of TAK1 and $\mathrm{TAB} 2$, it has been elucidated that TAK1 regulates angiogenesis by inhibiting the TNF-dependent death of endothelial cells and promoting TNF-independent angiogenic cell migration. TAK1 is suggested to modulate endothelial cell survival in a VEGF-independent manner and prevent TNF-induced cell death though the modulation of RIP1dependent necrosis [55].

Myeloid-specific deletion of TAK1 using the LysM-Cre transgene results in the spontaneous death of bone marrowderived macrophages (BMDMs) [56, 57]. Unlike previous data showing the prevention of keratinocyte death by simultaneous deletion of TNFR1, macrophage death was not rescued by the deletion of TNFR1 [47, 57]. TAK1 deficiency in $\mathrm{CD}_{11 \mathrm{c}^{+}}$DCs enhances apoptosis by increased caspase activity that depletes $\mathrm{CD} 8^{+}$and $\mathrm{CD} 103^{+}$ DC subsets in lymphoid and nonlymphoid tissues, respectively [58]. Moreover, TAK1 is a critical signaling intermediate in TLR-induced activation of NF- $\mathrm{KB}$ in DCs.

While TAK1 is known to have prosurvival and proinflammatory roles in most immune cells, recent studies have found novel negative regulatory functions of TAK1 in neutrophils [56]. The study demonstrated that myeloidspecific TAK1 deletion results in neutrophilia and myeloproliferative disorder (splenomegaly and lymphomegaly) $[56,57]$. In addition, LPS treatment of $\mathrm{TAK} 1^{-/-}$neutrophils increased the phosphorylation of IKK, JNK, and p38, leading to an enhanced production of proinflammatory cytokines (TNF- $\alpha$, IL-1 $\beta$ and IL-6) and ROS $[56,59]$.

After BCR ligation, the caspase recruitment domain family member 11 (CARD11/CARMA1)-B cell CLL/ lymphoma 10 (BCL10)-mucosa-associated, lymphoid tissue, lymphoma translocation gene 1 (MALT1) complex interacts with TRAF6, which in turn induces TAK1 activation [60]. Although TAK1 plays a role in cytokine and 
TLR-induced NF- $\mathrm{KB}$ and MAPK signaling in B cells, TAK1 contributes only to JNK activation in BCR signaling $[60,61]$. Moreover, TAK1 deficiency in B cells causes impaired development, thus validating that TAK1 is indispensable for B cell development. Furthermore, TAK1 is required for the survival and maintenance of peripheral $\mathrm{T}$ cells and for TCR-mediated NF- $\kappa \mathrm{B}$ and MAPK activation [62-64]. Deletion of TAK1 in mature thymocytes impairs TCR-mediated activation of NF- $\mathrm{KB}$ and JNK, and sensitizes cells to apoptosis. However, in effector T cells, TAK1 is dispensable for NF- $\kappa \mathrm{B}$ and JNK signaling, but is crucial for proliferation and p38 activation in response to TCR stimulation [64]. Thus, TAK1 is a critical component for cell homeostasis and acts as a regulator of NF- $\mathrm{\kappa B}$ and MAPK pathways in a cell type-specific and receptordependent manner. These functions are exerted through diverse pathways, including the regulation of inflammation, apoptosis, proliferation, and carcinogenesis.

\section{Dual role of TAK1 in cancer development}

TAK1 has become an attractive target for drug development due to its roles in inflammation, survival, and carcinogenesis. Various tissue-specific TAK1 knockout mouse models have elucidated novel functions of TAK1 in vivo. Increasing evidence shows the association of TAK1 mutation or loss with human cancers. TAK1 mutations were identified in patients with diffuse, large B-cell lymphoma [65]. Furthermore, frequent deletion of 6q15, including the $M A P 3 K 7$ gene that encodes TAK1 protein, is reported to be associated with the advanced stages of prostate cancer in humans [66]. The decrease of TAK1 expression with increasing Gleason scores in human prostate cancer specimens emphasizes that TAK1 is a tumor suppressor in prostate cancer [3]. The engraftment of $\mathrm{TAK}^{-1-}$ prostate stem cells in mice displayed features of prostatic intraepithelial neoplasia and invasive carcinoma [3]. Detailed mechanistic studies in mouse prostate cancer stem cells and TAK1-deficient prostate epithelial cells determined that TAK1 deletion enhances cell proliferation, migration, and invasion, and inhibits pro-apoptotic p38/ JNK MAPK signaling. While there exists an association between TAK1 mutation and certain cancer developments, Ray and others demonstrated that the inhibition of TAK1 prevents lung metastasis of breast cancer [67], suggesting the role of TAK1 in tumor progression, metastasis, and tumor microenvironment. Recent studies also demonstrate that TAK1 is associated with lymphoid malignancies. For example, activated TAK1 is abundantly observed in multiple lymphoma cell lines and the abrogation of TAK1 results in reduced NF- $\mathrm{\kappa B}$ and $\mathrm{p} 38$ activation that becomes vulnerable to apoptosis. Thus, TAK1 is essential for the maintenance of lymphoma survival and will be a target for lymphoma therapy [68]. Recently, it has been reported that TAK1 is important for the viability of cancer cells displaying hyperactive KRAS-dependent Wnt signaling, as the induction of TAK1 inhibition promoted apoptosis in these KRAS-dependent colon cancers, but not in KRASindependent colon cancers [69]. These reports suggest, again, the dual functions of TAK1 in tumor initiation, progression, and metastasis as either a tumor promoter or suppressor, being context-dependent and cell type-dependent.

\section{Targeting TAK1 for the treatment of cancer}

It is becoming increasingly clear that proinflammatory cytokines such as TNF- $\alpha$, IL- $1 \beta$, and IL- 6 can promote cancer development and progression [70]. The tumor microenvironment contributes to the link between chronic inflammation and cancer, which promotes tumor progression. It is necessary to elucidate the physiological functions of TAK1 in different cell types in chronic inflammation and cancer. Investigation of other regulators has shed more light on the role of TAK1 in the development of inflammation-associated cancer. Genetic deletion of CYLD or ITCH, essential ubiquitin regulators for TAK1 deactivation, causes strong and sustained TAK1 activation with enhanced production of tumor-promoting proinflammatory cytokines that mediate liver fibrosis, tumor development, and metastasis $[26,71]$. By contrast, TAK $1^{-/-}$neutrophils increase activation of IKK, JNK, and p38, leading to the enhanced production of proinflammatory cytokines and ROS. Given that neutrophils possess a significant impact on tumor immune surveillance, metastasis, angiogenesis, and cellular proliferation, targeting TAK1 activity in neutrophils can be a therapeutic strategy for cancer treatment through the regulation of cancer-associated inflammation and the tumor microenvironment.

Genotoxic agents induce TAK1-mediated NF- $\kappa B$ activation, suggesting that TAK1 is associated with resistance to cancer chemotherapy. The first investigation of reduced chemoresistance by TAK1 inhibition was reported for human pancreatic cancer. The utilization of RNAi-mediated silencing of TAK1 as well as LYTAK1, an orally active TAK1 inhibitor, significantly inhibited NF- $\mathrm{KB}$ activity and sensitized cancer cells to gemcitabine, oxaliplatin, and SN38 [4]. LYTAK1 in combination with gemcitabine reduced tumor volume and prolonged survival in vivo. The efficacy of the topoisomerase inhibitor camptothecin was enhanced by the RNAi silencing of TAK1 in human breast and colon cancer cells, but not in normal mammary epithelial cells [5].

The extrinsic apoptotic signaling induced by TNF-related apoptosis-inducing ligand (TRAIL), a promising target 
for cancer therapy, was enhanced by RNAi silencing and chemical inhibition of TAK1 by suppressing NF- $\mathrm{KB}$ and AMPK-dependent cytoprotective autophagy [72, 73]. Epidermal growth factor receptor (EGFR) is known to promote cancer progression and is considered to be a therapeutic target in various cancers. EGFR-mediated p38 activation inhibits TAK1 activation through TAB1 phosphorylation [74]. Simultaneously, EGFR signaling induces prosurvival signaling through TAK1-p38 [75]. Thus, targeting the crosstalk between TAK1 and EGFR signaling may be a powerful strategy for cancer therapy [75]. TAK1 is also associated with the pathways of AMPK, Snf1, and LKB1 that control tumor metabolism and development [73].

\section{Conclusion and future perspectives}

In recent years, cell-specific functions of TAK1 have significantly progressed towards elucidation. TAK1 can be activated by various stimuli, including TLR ligands and cytokines. The ubiquitination and phosphorylation of TAK1 and its associated partners (TAB1, TAB2, and TAB3) are central for the activation of downstream NF- $\kappa B$, JNK, p38, and ERK signaling pathways. Because of the embryonic lethality of whole-body TAK1 deletion, the specific function of TAK1 has been studied using cell- or tissue-specific TAK1 knockouts. Studies from animal models suggest that TAK1 exhibits diverse and complex biological functions in regulating cell survival, inflammation, and tumorigenesis. These findings suggest that TAK1 is now an attractive molecular target for the treatment of several human diseases. Systemic administration of selective chemical TAK1 inhibitors showed significant antiinflammatory and cancer therapeutic activities in animal models; however, the fact that TAK1 has diverse cellular functions increases the risk of undesirable side effects, especially the susceptibility to bacterial and viral infections. In addition, it must be cautioned that the deletion of TAK1 in hepatocytes results in spontaneous hepatic injury, inflammation, fibrosis, and cancer through the TNFR and TGF $\beta R$ signaling or NF- $\kappa B$-independent function of NEMO [2, 40]. Unfortunately, our knowledge on when and where TAK1 is activated in pathologic tissues is insufficient. For a better understanding of TAK1, the establishment of proper methods (i.e., immunohistochemistry) for the detection of the TAK1 activity will provide valuable information about its pathophysiological relevance.

RNAi-mediated silencing of TAK1 can ameliorate inflammation in experimental autoimmune arthritis with the decreased frequency of Th1 and Th17 cell infiltrations in mice [76]. The RNAi-mediated strategy is also promising in the approach to silence other TAK1-associated targets involved in the TAK1 complex, including TABs and TRAFs. In addition, future characterization of ubiquitin-related enzymes for TAK1 activation and inactivation could provide new strategies for targeting TAK1 in liver disease.

Acknowledgments This study was supported by NIH grants R01AA02172, R01DK085252, P42 ES010337, and by the pilot grant from the UCSD Digestive Diseases Research Development Center (DK080506).

Conflict of interest The authors declare that they have no conflict of interest.

\section{References}

1. Yamaguchi K, Shirakabe K, Shibuya H, Irie K, Oishi I, Ueno N, et al. Identification of a member of the MAPKKK family as a potential mediator of TGF-beta signal transduction. Science. 1995;270(5244):2008-11.

2. Inokuchi S, Aoyama T, Miura K, Osterreicher CH, Kodama Y, Miyai K, et al. Disruption of TAK1 in hepatocytes causes hepatic injury, inflammation, fibrosis, and carcinogenesis. Proc Natl Acad Sci USA. 2010;107(2):844-9.

3. Wu M, Shi L, Cimic A, Romero L, Sui G, Lees CJ, et al. Suppression of Tak1 promotes prostate tumorigenesis. Cancer Res. 2012;72(11):2833-43.

4. Melisi D, Xia Q, Paradiso G, Ling J, Moccia T, Carbone C, et al. Modulation of pancreatic cancer chemoresistance by inhibition of TAK1. J Natl Cancer Inst. 2011;103(15):1190-204.

5. Martin SE, Wu ZH, Gehlhaus K, Jones TL, Zhang YW, Guha R, et al. RNAi screening identifies TAK1 as a potential target for the enhanced efficacy of topoisomerase inhibitors. Curr Cancer Drug Targets. 2011;11(8):976-86.

6. Shibuya H, Yamaguchi K, Shirakabe K, Tonegawa A, Gotoh Y, Ueno N, et al. TAB1: an activator of the TAK1 MAPKKK in TGF-beta signal transduction. Science. 1996;272(5265):1179-82.

7. Ishitani T, Takaesu G, Ninomiya-Tsuji J, Shibuya H, Gaynor RB, Matsumoto K. Role of the TAB2-related protein TAB3 in IL-1 and TNF signaling. EMBO J. 2003;22(23):6277-88.

8. Cheung PC, Nebreda AR, Cohen P. TAB3, a new binding partner of the protein kinase TAK1. Biochem J. 2004;378(Pt 1):27-34.

9. Shim JH, Xiao C, Paschal AE, Bailey ST, Rao P, Hayden MS, et al. TAK1, but not TAB1 or TAB2, plays an essential role in multiple signaling pathways in vivo. Genes Dev. 2005;19(22): 2668-81.

10. Komatsu Y, Shibuya H, Takeda N, Ninomiya-Tsuji J, Yasui T, Miyado K, et al. Targeted disruption of the Tab1 gene causes embryonic lethality and defects in cardiovascular and lung morphogenesis. Mech Dev. 2002;119(2):239-49.

11. Sanjo H, Takeda K, Tsujimura T, Ninomiya-Tsuji J, Matsumoto $\mathrm{K}$, Akira S. TAB2 is essential for prevention of apoptosis in fetal liver but not for interleukin-1 signaling. Mol Cell Biol. 2003;23(4):1231-8.

12. Singhirunnusorn P, Suzuki S, Kawasaki N, Saiki I, Sakurai H. Critical roles of threonine 187 phosphorylation in cellular stressinduced rapid and transient activation of transforming growth factor-beta-activated kinase 1 (TAK1) in a signaling complex containing TAK1-binding protein TAB1 and TAB2. J Biol Chem. 2005;280(8):7359-68.

13. Pathak S, Borodkin VS, Albarbarawi O, Campbell DG, Ibrahim A, van Aalten DM. O-GlcNAcylation of TAB1 modulates TAK1mediated cytokine release. EMBO J. 2012;31(6):1394-404. 
14. Takaesu G, Surabhi RM, Park KJ, Ninomiya-Tsuji J, Matsumoto $\mathrm{K}$, Gaynor RB. TAK1 is critical for IkappaB kinase-mediated activation of the NF-kappaB pathway. J Mol Biol. 2003;326(1): $105-15$.

15. Ninomiya-Tsuji J, Kishimoto K, Hiyama A, Inoue J, Cao Z, Matsumoto K. The kinase TAK1 can activate the NIK-I kappaB as well as the MAP kinase cascade in the IL-1 signalling pathway. Nature. 1999;398(6724):252-6.

16. Sakurai H, Miyoshi H, Toriumi W, Sugita T. Functional interactions of transforming growth factor beta-activated kinase 1 with IkappaB kinases to stimulate NF-kappaB activation. J Biol Chem. 1999;274(15):10641-8.

17. Song HY, Regnier CH, Kirschning CJ, Goeddel DV, Rothe M. Tumor necrosis factor (TNF)-mediated kinase cascades: bifurcation of nuclear factor-kappaB and c-jun $\mathrm{N}$-terminal kinase (JNK/SAPK) pathways at TNF receptor-associated factor 2. Proc Natl Acad Sci USA. 1997;94(18):9792-6.

18. Adhikari A, Xu M, Chen ZJ. Ubiquitin-mediated activation of TAK1 and IKK. Oncogene. 2007;26(22):3214-26.

19. Seki E, Brenner DA, Karin M. A liver full of JNK: signaling in regulation of cell function and disease pathogenesis, and clinical approaches. Gastroenterology. 2012;143(2):307-20.

20. Yamashita M, Fatyol K, Jin C, Wang X, Liu Z, Zhang YE. TRAF6 mediates Smad-independent activation of JNK and p38 by TGF-beta. Mol Cell. 2008;31(6):918-24.

21. Kanayama A, Seth RB, Sun L, Ea CK, Hong M, Shaito A, et al. $\mathrm{TAB} 2$ and TAB3 activate the NF-kappaB pathway through binding to polyubiquitin chains. Mol Cell. 2004;15(4):535-48.

22. Broglie P, Matsumoto K, Akira S, Brautigan DL, Ninomiya-Tsuji J. Transforming growth factor beta-activated kinase 1 (TAK1) kinase adaptor, TAK1-binding protein 2 , plays dual roles in TAK1 signaling by recruiting both an activator and an inhibitor of TAK1 kinase in tumor necrosis factor signaling pathway. J Biol Chem. 2010;285(4):2333-9.

23. Kajino T, Ren H, Iemura S, Natsume T, Stefansson B, Brautigan DL, et al. Protein phosphatase 6 down-regulates TAK1 kinase activation in the IL-1 signaling pathway. J Biol Chem. 2006;281(52):39891-6.

24. Cheung PC, Campbell DG, Nebreda AR, Cohen P. Feedback control of the protein kinase TAK1 by SAPK2a/p38alpha. EMBO J. 2003;22(21):5793-805.

25. Courtois G. Tumor suppressor CYLD: negative regulation of NFkappaB signaling and more. Cell Mol Life Sci. 2008;65(7-8): 1123-32.

26. Ahmed N, Zeng M, Sinha I, Polin L, Wei WZ, Rathinam C, et al. The E3 ligase Itch and deubiquitinase Cyld act together to regulate Tak1 and inflammation. Nat Immunol. 2011;12(12):1176-83.

27. Fan Y, Shi Y, Liu S, Mao R, An L, Zhao Y, et al. Lys48-linked TAK1 polyubiquitination at lysine-72 downregulates TNFalphainduced NF-kappaB activation via mediating TAK1 degradation. Cell Signal. 2012;24(7):1381-9.

28. Ruland J. Return to homeostasis: downregulation of NF-kappaB responses. Nat Immunol. 2011;12(8):709-14.

29. Kim SI, Kwak JH, Na HJ, Kim JK, Ding Y, Choi ME. Transforming growth factor-beta (TGF-beta1) activates TAK1 via TAB1-mediated autophosphorylation, independent of TGF-beta receptor kinase activity in mesangial cells. J Biol Chem. 2009;284(33):22285-96.

30. Watkins SJ, Jonker L, Arthur HM. A direct interaction between TGFbeta activated kinase 1 and the TGFbeta type II receptor: implications for TGFbeta signalling and cardiac hypertrophy. Cardiovasc Res. 2006;69(2):432-9.

31. Sano Y, Harada J, Tashiro S, Gotoh-Mandeville R, Maekawa T, Ishii S. ATF-2 is a common nuclear target of Smad and TAK1 pathways in transforming growth factor-beta signaling. J Biol Chem. 1999;274(13):8949-57.
32. Jadrich JL, O'Connor MB, Coucouvanis E. The TGF beta activated kinase TAK1 regulates vascular development in vivo. Development. 2006;133(8):1529-41.

33. Sorrentino A, Thakur N, Grimsby S, Marcusson A, von Bulow V, Schuster N, et al. The type I TGF-beta receptor engages TRAF6 to activate TAK1 in a receptor kinase-independent manner. Nat Cell Biol. 2008;10(10):1199-207.

34. Xia ZP, Sun L, Chen X, Pineda G, Jiang X, Adhikari A, et al. Direct activation of protein kinases by unanchored polyubiquitin chains. Nature. 2009;461(7260):114-9.

35. Mao R, Fan Y, Mou Y, Zhang H, Fu S, Yang J. TAK1 lysine 158 is required for TGF-beta-induced TRAF6-mediated Smad-independent IKK/NF-kappaB and JNK/AP-1 activation. Cell Signal. 2011;23(1):222-7.

36. Wu ZH, Wong ET, Shi Y, Niu J, Chen Z, Miyamoto S, et al. ATM- and NEMO-dependent ELKS ubiquitination coordinates TAK1-mediated IKK activation in response to genotoxic stress. Mol Cell. 2010;40(1):75-86.

37. Yang Y, Xia F, Hermance N, Mabb A, Simonson S, Morrissey S, et al. A cytosolic ATM/NEMO/RIP1 complex recruits TAK1 to mediate the NF-kappaB and p38 mitogen-activated protein kinase (MAPK)/MAPK-activated protein 2 responses to DNA damage. Mol Cell Biol. 2011;31(14):2774-86.

38. Hinz M, Stilmann M, Arslan SC, Khanna KK, Dittmar G, Scheidereit C. A cytoplasmic ATM-TRAF6-cIAP1 module links nuclear DNA damage signaling to ubiquitin-mediated NF-kappaB activation. Mol Cell. 2010;40(1):63-74.

39. Niu J, Shi Y, Iwai K, Wu ZH. LUBAC regulates NF-kappaB activation upon genotoxic stress by promoting linear ubiquitination of NEMO. EMBO J. 2011;30(18):3741-53.

40. Bettermann K, Vucur M, Haybaeck J, Koppe C, Janssen J, Heymann F, et al. TAK1 suppresses a NEMO-dependent but NFkappaB-independent pathway to liver cancer. Cancer Cell. 2010;17(5):481-96.

41. Beg AA, Baltimore D. An essential role for NF-kappaB in preventing TNF-alpha-induced cell death. Science. 1996;274(5288):782-4.

42. Van Antwerp DJ, Martin SJ, Kafri T, Green DR, Verma IM. Suppression of TNF-alpha-induced apoptosis by NF-kappaB. Science. 1996;274(5288):787-9.

43. Schwabe RF, Brenner DA. Mechanisms of liver injury. I. TNFalpha-induced liver injury: role of IKK, JNK, and ROS pathways. Am J Physiol Gastrointest Liver Physiol. 2006;290(4):G583-9.

44. Luedde T, Beraza N, Kotsikoris V, van Loo G, Nenci A, De Vos $\mathrm{R}$, et al. Deletion of NEMO/IKKgamma in liver parenchymal cells causes steatohepatitis and hepatocellular carcinoma. Cancer Cell. 2007;11(2):119-32.

45. Yang L, Inokuchi S, Roh YS, Song J, Loomba R, Park EJ, et al. Transforming growth factor-beta signaling in hepatocytes promotes hepatic fibrosis and carcinogenesis in mice with hepatocyte-specific deletion of TAK1. Gastroenterology. 2013;144(5): 1042-1054 e4.

46. Yang L, Roh YS, Song J, Zhang B, Liu C, Loomba R, et al. TGFbeta signaling in hepatocytes participates in steatohepatitis through regulation of cell death and lipid metabolism. Hepatology. 2013.

47. Omori E, Matsumoto K, Sanjo H, Sato S, Akira S, Smart RC, et al. TAK1 is a master regulator of epidermal homeostasis involving skin inflammation and apoptosis. $\mathrm{J}$ Biol Chem. 2006;281(28):19610-7.

48. Kajino-Sakamoto R, Omori E, Nighot PK, Blikslager AT, Matsumoto K, Ninomiya-Tsuji J. TGF-beta-activated kinase 1 signaling maintains intestinal integrity by preventing accumulation of reactive oxygen species in the intestinal epithelium. J Immunol. 2010;185(8):4729-37.

49. Omori E, Morioka S, Matsumoto K, Ninomiya-Tsuji J. TAK1 regulates reactive oxygen species and cell death in keratinocytes, 
which is essential for skin integrity. J Biol Chem. 2008;283(38): 26161-8.

50. Omori E, Inagaki M, Mishina Y, Matsumoto K, Ninomiya-Tsuji J. Epithelial transforming growth factor beta-activated kinase 1 (TAK1) is activated through two independent mechanisms and regulates reactive oxygen species. Proc Natl Acad Sci USA. 2012;109(9):3365-70.

51. Kajino-Sakamoto R, Inagaki M, Lippert E, Akira S, Robine S, Matsumoto K, et al. Enterocyte-derived TAK1 signaling prevents epithelium apoptosis and the development of ileitis and colitis. J Immunol. 2008;181(2):1143-52.

52. Vanlangenakker N, Vanden Berghe T, Bogaert P, Laukens B, Zobel K, Deshayes K, et al. cIAP1 and TAK1 protect cells from TNF-induced necrosis by preventing RIP1/RIP3-dependent reactive oxygen species production. Cell Death Differ. 2011;18(4):656-65.

53. Dondelinger Y, Aguileta MA, Goossens V, Dubuisson C, Grootjans S, Dejardin E, et al. RIPK3 contributes to TNFR1mediated RIPK1 kinase-dependent apoptosis in conditions of cIAP1/2 depletion or TAK1 kinase inhibition. Cell Death Differ. 2013;20(10):1381-92.

54. Shim JH, Greenblatt MB, Xie M, Schneider MD, Zou W, Zhai B, et al. TAK1 is an essential regulator of BMP signalling in cartilage. EMBO J. 2009;28(14):2028-41.

55. Morioka S, Inagaki M, Komatsu Y, Mishina Y, Matsumoto K, Ninomiya-Tsuji J. TAK1 kinase signaling regulates embryonic angiogenesis by modulating endothelial cell survival and migration. Blood. 2012;120(18):3846-57.

56. Ajibade AA, Wang Q, Cui J, Zou J, Xia X, Wang M, et al. TAK1 negatively regulates NF-kappaB and p38 MAP kinase activation in Gr-1+CD11b+ neutrophils. Immunity. 2012;36(1):43-54.

57. Lamothe B, Lai Y, Hur L, Orozco NM, Wang J, Campos AD, et al. Deletion of TAK1 in the myeloid lineage results in the spontaneous development of myelomonocytic leukemia in mice. PLoS ONE. 2012;7(12):e51228.

58. Wang Y, Huang G, Vogel P, Neale G, Reizis B, Chi H. Transforming growth factor beta-activated kinase 1 (TAK1)-dependent checkpoint in the survival of dendritic cells promotes immune homeostasis and function. Proc Natl Acad Sci USA. 2012;109(6): E343-52.

59. Eftychi C, Karagianni N, Alexiou M, Apostolaki M, Kollias G. Myeloid TAKI [corrected] acts as a negative regulator of the LPS response and mediates resistance to endotoxemia. PLoS ONE. 2012;7(2):e31550.

60. Sato S, Sanjo H, Takeda K, Ninomiya-Tsuji J, Yamamoto M, Kawai T, et al. Essential function for the kinase TAK1 in innate and adaptive immune responses. Nat Immunol. 2005;6(11): 1087-95.

61. Schuman J, Chen Y, Podd A, Yu M, Liu HH, Wen R, et al. A critical role of TAK1 in B-cell receptor-mediated nuclear factor kappaB activation. Blood. 2009;113(19):4566-74.

62. Liu HH, Xie M, Schneider MD, Chen ZJ. Essential role of TAK1 in thymocyte development and activation. Proc Natl Acad Sci USA. 2006;103(31):11677-82.

63. Sato S, Sanjo H, Tsujimura T, Ninomiya-Tsuji J, Yamamoto M, Kawai T, et al. TAK1 is indispensable for development of T cells and prevention of colitis by the generation of regulatory $\mathrm{T}$ cells. Int Immunol. 2006;18(10):1405-11.

64. Wan YY, Chi H, Xie M, Schneider MD, Flavell RA. The kinase TAK1 integrates antigen and cytokine receptor signaling for $\mathrm{T}$ cell development, survival and function. Nat Immunol. 2006;7(8):851-8.

65. Compagno M, Lim WK, Grunn A, Nandula SV, Brahmachary M, Shen Q, et al. Mutations of multiple genes cause deregulation of NF-kappaB in diffuse large B-cell lymphoma. Nature. 2009;459(7247):717-21.

66. Liu W, Chang BL, Cramer S, Koty PP, Li T, Sun J, et al. Deletion of a small consensus region at $6 \mathrm{q} 15$, including the MAP3K7 gene, is significantly associated with high-grade prostate cancers. Clin Cancer Res. 2007;13(17):5028-33.

67. Ray DM, Myers PH, Painter JT, Hoenerhoff MJ, Olden K, Roberts JD. Inhibition of transforming growth factor-beta-activated kinase-1 blocks cancer cell adhesion, invasion, and metastasis. Br J Cancer. 2012;107(1):129-36.

68. Buglio D, Palakurthi S, Byth K, Vega F, Toader D, Saeh J, et al. Essential role of TAK1 in regulating mantle cell lymphoma survival. Blood. 2012;120(2):347-55.

69. Singh A, Sweeney MF, Yu M, Burger A, Greninger P, Benes C, et al. TAK1 inhibition promotes apoptosis in KRAS-dependent colon cancers. Cell. 2012;148(4):639-50.

70. Karin M. Nuclear factor-kappaB in cancer development and progression. Nature. 2006;441(7092):431-6.

71. Nikolaou K, Tsagaratou A, Eftychi C, Kollias G, Mosialos G, Talianidis I. Inactivation of the deubiquitinase CYLD in hepatocytes causes apoptosis, inflammation, fibrosis, and cancer. Cancer Cell. 2012;21(6):738-50.

72. Choo MK, Kawasaki N, Singhirunnusorn P, Koizumi K, Sato S, Akira $\mathrm{S}$, et al. Blockade of transforming growth factor-betaactivated kinase 1 activity enhances TRAIL-induced apoptosis through activation of a caspase cascade. Mol Cancer Ther. 2006;5(12):2970-6.

73. Herrero-Martin G, Hoyer-Hansen M, Garcia-Garcia C, Fumarola C, Farkas T, Lopez-Rivas A, et al. TAK1 activates AMPKdependent cytoprotective autophagy in TRAIL-treated epithelial cells. EMBO J. 2009;28(6):677-85.

74. Shin MS, Shinghirunnusorn P, Sugishima Y, Nishimura M, Suzuki S, Koizumi $\mathrm{K}$, et al. Cross interference with TNF-alphainduced TAK1 activation via EGFR-mediated p38 phosphorylation of TAK1-binding protein 1. Biochim Biophys Acta. 2009;1793(7):1156-64.

75. Nishimura M, Shin MS, Singhirunnusorn P, Suzuki S, Kawanishi M, Koizumi K, et al. TAK1-mediated serine/threonine phosphorylation of epidermal growth factor receptor via p38/extracellular signal-regulated kinase: NF-\{kappa\}B-independent survival pathways in tumor necrosis factor alpha signaling. Mol Cell Biol. 2009;29(20):5529-39.

76. Courties G, Seiffart V, Presumey J, Escriou V, Scherman D, Zwerina $\mathrm{J}$, et al. In vivo RNAi-mediated silencing of TAK1 decreases inflammatory Th1 and Th17 cells through targeting of myeloid cells. Blood. 2010;116(18):3505-16. 